Article

\title{
A Design of Finite Memory Residual Generation Filter for Sensor Fault Detection
}

\author{
Pyung Soo Kim \\ Department of Electronic Engineering, Korea Polytechnic University, Siheung-si, Gyeonggi-do, 15073, Korea \\ * Correspondence: pspeter.kim@gmail.com; pskim@kpu.ac.kr; Tel.: +82-31-8041-0489
}

\begin{abstract}
In the current paper, a residual generation filter with finite memory structure is proposed for sensor fault detection. The proposed finite memory residual generation filter provides the residual by real-time filtering of fault vector using only the most recent finite observations and inputs on the window. It is shown that the residual given by the proposed residual generation filter provides the exact fault for noise-free systems. The proposed residual generation filter is specified to the digital filter structure for the amenability to hardware implementation. Finally, to illustrate the capability of the proposed residual generation filter, numerical examples are performed for the discretized DC motor system having the multiple sensor faults.
\end{abstract}

Keywords: residual generation filter; finite memory structure; Kalman filter; fast detection

\section{Introduction}

As most dynamic process plants are becoming more complex, there has been a growing demand for a fault detection. The fault detection is the prompt indication of incipient as well as abrupt faults and can help avoid major plant breakdowns and take appropriate actions in order to maintain the operation. Thus, a fault detection is an important and challenging problem in many disciplines such as chemical engineering, nuclear engineering, aerospace engineering, and automotive systems [1]-[3].

The essential step for the fault detection is to generate a set of variables known as residuals by using one or more residual generation filters. These residuals should ideally be zero (or zero mean) under no-fault conditions. In practical applications, the residuals are corrupted by the presence of noise, unknown disturbances, and uncertainties in the system model. Hence, in order to be useful in practical applications, they should be insensitive to noise, disturbances, and model uncertainties while maximally sensitive to faults.

As residual generation filters, Kalman filters have been adopted in the stochastic case where noises have to be considered [4]-[9]. Due to the compact representation and the efficient manner, the Kalman filter has been applied successfully for various areas including a fault detection. However, the Kalman filter has an infinite memory structure that utilizes all observations accomplished by equaling weighting and has a recursive formulation. Thus, the Kalman filter tends to accumulate the filtering error as time goes and can show even divergence phenomenon for temporary modeling uncertainties and round-off errors [10]-[13]. This inherent property of the Kalman filter has been shown in applications of wireless sensor networks [14][15]. In addition, actually, long past measurements are not useful for detection of faults with unknown times of occurrence. Moreover, it is also known that the increase of the number of measurements for a detection decision will increase detection latency in a system for detecting a signal with unknown time of occurrence.

Therefore, in the current paper, an alternative residual generation filter with finite memory structure is proposed for a sensor fault detection. The proposed finite memory residual generation filter provides the residual by real-time filtering of fault vector using only the most recent finite observations and inputs on the window. It is shown that the residual given by the proposed residual generation filter provides the exact fault for noise-free systems. The proposed residual generation filter is specified to the digital filter structure for the amenability to hardware implementation. Finally, numerical examples are performed for the discretized DC motor system having the multiple sensor faults to illustrate the capability of the proposed finite memory residual generation filter. 


\section{Problem Statements}

In general, the fault model can be represented by the following discrete-time state space model with unknown sensor faults as well as noises:

$$
\begin{aligned}
x(i+1) & =\Phi x(i)+D u(i)+\Delta_{1} f(i)+E \omega(i), \\
y(i) & =C x(i)+\Delta_{2} f(i)+v(i)
\end{aligned}
$$

where $x(i) \in \Re^{n}$ the state vector, $u(i) \in \Re^{l}$ and $y(i) \in \Re^{q}$ are the input vector and the measurement vector. The covariances of the system noise $\omega(i) \in \Re^{p}$ and the measurement noise $v(i) \in \Re^{q}$ are $Q_{\omega}$ and $R$, respectively. The fault vector $\omega(i) \in \Re^{q}$ in the system under consideration are to be represented by random-walk processes as

$$
f(i+1)=f(i)+\delta(i)
$$

where $f(i)=\left[f_{1}(i) f_{2}(i) \cdots f_{q}(i)\right]^{T}$, and $\delta(i)=\left[\delta_{1}(i) \delta_{2}(i) \cdots \delta_{q}(i)\right]^{T}$ is a zero-mean white Gaussian random process with covariance $Q_{\delta}$. It is noted that the random-walk process provides a general and useful tool for the analysis of unknown time-varying parameters and has been widely used in the detection and estimation area.

The fault model (1) can be rewritten as an augmented state model as

$$
\begin{aligned}
{\left[\begin{array}{c}
x(i+1) \\
f(i+1)
\end{array}\right] } & =A\left[\begin{array}{l}
x(i) \\
f(i)
\end{array}\right]+B u(i)+G\left[\begin{array}{c}
\omega(i) \\
\delta(i)
\end{array}\right], \\
y(i) & =H\left[\begin{array}{l}
x(i) \\
f(i)
\end{array}\right]+v(i)
\end{aligned}
$$

where

$$
A=\left[\begin{array}{cc}
\Phi & \Delta_{1} \\
0 & I
\end{array}\right], B=\left[\begin{array}{c}
D \\
0
\end{array}\right], G=\left[\begin{array}{cc}
E & 0 \\
0 & I
\end{array}\right], H=\left[\begin{array}{ll}
C & \Delta_{2}
\end{array}\right],
$$

and the covariance $w(i)$ is the diagonal matrix with $Q_{\omega}$ and $Q_{\delta}$.

In general, the residual for the fault detection is defined by a signal generated based on the measurement vector $y(i)$ and input vector $u(i)$. In addition, this residual is corrupted by the presence of noises in practical applications. Therefore, a residual generation filter is required to generate robust residuals that are insensitive to these noises, while sensitive to faults.

In the current paper, an alternative residual generation filter is designed with finite memory structure. The proposed finite memory residual generation filter provides the residual $r(i)$ by real-time filtering of fault vector $f(i)$ using only the most recent finite measurements $Y(i)$ and inputs $U(i)$ on the window $[i-M, i]$ as follows

$$
r(i) \triangleq \hat{f}(i) \triangleq \mathcal{H}[Y(i)-\Omega U(i)]
$$

where $\hat{f}(i)$ is the filtered estimate of $f(i)$ and $\mathcal{H}$ is the filter gain matrix. The term $Y(i)-\Omega U(i)$ in (4) with the most recent finite measurements $Y(i)$ and inputs $U(i)$ can be represented by the following regression form on the window $[i-M, i]$ :

$$
Y(i)-\Omega U(i)=\Gamma\left[\begin{array}{l}
x(i) \\
f(i)
\end{array}\right]+\Lambda W(i)+V(i)
$$


where

$$
Y(i) \triangleq\left[y^{T}(i-M) y^{T}(i-M+1) \cdots y^{T}(i-1)\right]^{T}
$$

and $U(i), W(i), V(i)$ have the same form as (6) for $u(i), w(i), v(i)$, respectively, and matrices $\Omega, \Gamma, \Lambda$ are as follows:

$$
\begin{aligned}
& \Omega \triangleq-\left[\begin{array}{cccc}
H A^{-1} B & H A^{-2} B & \cdots & H A^{-M} B \\
0 & H A^{-1} B & \cdots & H A^{-M+1} B \\
\vdots & \vdots & \vdots & \vdots \\
0 & 0 & \cdots & H A^{-1} B
\end{array}\right], \Gamma \triangleq\left[\begin{array}{c}
H A^{-M} \\
H A^{-M+1} \\
\vdots \\
H A^{-1}
\end{array}\right], \\
& \Lambda \triangleq-\left[\begin{array}{cccc}
H A^{-1} G & H A^{-2} G & \cdots & H A^{-M} G \\
0 & H A^{-1} G & \cdots & H A^{-M+1} G \\
\vdots & \vdots & \vdots & \vdots \\
0 & 0 & \cdots & H A^{-1} G
\end{array}\right] .
\end{aligned}
$$

The noise term $\Lambda W(i)+V(i)$ in (5) is zero-mean white Gaussian with covariance $\Pi$ given by

$$
\Pi \triangleq \Lambda[\operatorname{diag}(\overbrace{Q Q \cdots Q}^{M})] \Lambda^{T}+[\operatorname{diag}(\overbrace{R R \cdots R}^{M})] .
$$

Now, to get the residual generation filter from the regression form (5), the following weighted least square cost function must be minimized:

$$
\left\{Y(i)-\Omega U(i)-\Gamma\left[\begin{array}{l}
x(i) \\
f(i)
\end{array}\right]\right\}^{T} \Pi^{-1}\left\{\left[Y(i)-\Omega U(i)-\Gamma\left[\begin{array}{l}
x(i) \\
f(i)
\end{array}\right]\right\} .\right.
$$

Taking a derivation of (10) with respect to $\left[x^{T}(i) f^{T}(i)\right]^{T}$ and setting it to zero, the filter gain matrix $\mathcal{H}$ for the residual generation filter $r(i)=\hat{f}(i)$ is given by

$$
\mathcal{H}=\left[\left(\Gamma^{T} \Pi^{-1} \Gamma\right)^{-1} \Gamma^{T} \Pi^{-1}\right]_{q}
$$

where the subscript $q$ means the lower $q$ rows of $\left[\left(\Gamma^{T} \Pi^{-1} \Gamma\right)^{-1} \Gamma^{T} \Pi^{-1}\right]$. Therefore, the proposed finite memory residual generation filter for $r(i)$ is given by the simple matrix form with $\mathcal{H}$ and $Y(i)-\Omega U(i)$ as follows:

$$
\begin{aligned}
r(i) & =\left[\begin{array}{c}
r_{1}(i) \\
r_{2}(i) \\
\vdots \\
r_{q}(i)
\end{array}\right] \\
& =\left[\left(\Gamma^{T} \Pi^{-1} \Gamma\right)^{-1} \Gamma^{T} \Pi^{-1}\right]_{q}[Y(i)-\Omega U(i)] .
\end{aligned}
$$

Each residual $r_{s}(i)$ in the residual $r(i)$ can be obtained by

$$
r_{s}(i)=\mathcal{H}_{s}[Y(i)-\Omega U(i)]
$$

where $s=1,2, \cdots, q$ and $\mathcal{H}_{s}$ is the $s^{\text {th }}$ row of the $\mathcal{H}$.

The proposed finite memory residual generation filter for $r(i)$ in (12) is shown to have several inherent properties. As shown in the following Theorem, the residual $r(i)$ given by the proposed 
residual generation filter on the window $[i-M, i]$ provides the exact fault $f(i)$ when there are no noises.

Theorem 3.1. When $M \geq n$, the residual $r(i)$ given by the proposed finite memory residual generation filter on the window $[i-M, i]$ provides the exact fault $f(i)$ for noise-free systems. Proof:

When there are no noises on the window $[i-M, i]$ for the discrete-time state space model (2) with measurement delay as follows:

$$
\begin{aligned}
{\left[\begin{array}{l}
x(i+1) \\
f(i+1)
\end{array}\right] } & =A\left[\begin{array}{l}
x(i) \\
f(i)
\end{array}\right], \\
y(i) & =H\left[\begin{array}{l}
x(i) \\
f(i)
\end{array}\right],
\end{aligned}
$$

the measurements $Y(i)$ is determined from (5) as follows:

$$
Y(i)-\Omega U(i)=\Gamma\left[\begin{array}{l}
x(i) \\
f(i)
\end{array}\right] .
$$

Therefore, the following is true:

$$
\begin{aligned}
{\left[\begin{array}{c}
\hat{x}(i) \\
\hat{f}(i)
\end{array}\right] } & =\mathcal{H}[Y(i)-\Omega U(i)] \\
& =\left[\left(\Gamma^{T} \Pi^{-1} \Gamma\right)^{-1} \Gamma^{T} \Pi^{-1}\right][Y(i)-\Omega U(i)] \\
& =\left[\left(\Gamma^{T} \Pi^{-1} \Gamma\right)^{-1} \Gamma^{T} \Pi^{-1} \Gamma\right]\left[\begin{array}{c}
x(i) \\
f(i)
\end{array}\right] \\
& =\left[\begin{array}{l}
x(i) \\
f(i)
\end{array}\right] .
\end{aligned}
$$

This means that $r(i)=\hat{f}(i)=f(i)$.

Theorem 1 means that the residual $r(i)$ tracks exactly its actual fault $f(i)$ at every time for noise-free systems although the proposed finite memory residual generation filter has been designed assuming that the system (14) has additive system and measurement noises, $w(i)$ and $v(i)$ as the fault model (1). This property indicates finite convergence time and fast tracking ability of the residual given by the proposed residual generation filter. Thus, it can be expected that the proposed residual generation filter might be appropriate for fast detection.

The window length $M$ can be a useful design parameter for the proposed finite memory residual generation filter. Thus, the important issue here is how to choose an appropriate window length $M$ to make the residual performance as good as possible. The noise suppression of the proposed residual generation filter might be closely related to the window length $M$. It can have greater noise suppression as the window length $M$ increases, which improves the residual performance. However, as the window length increases, the convergence time of a filtered residual becomes long. This illustrates the proposed finite memory residual generation filter's compromise between the noise suppression and the tracking ability. Since $M$ is an integer, fine adjustment of the properties with $M$ is difficult. Moreover, it is difficult to determine the window length using systematic ways. In applications, one way to determine the window length is to take the appropriate value that can provide enough noise suppression. Therefore, it can be stated from above discussions that both the window length $M$ can be considered as a useful parameters to make the residual performance of the proposed finite memory residual generation filter as good as possible. 


\section{Specified Residual for Hardware Implementation}

In practice, it should be required that a fault detection system can be implemented with discrete-time analog or digital hardware. In this case, the fault detection system should be specified to an algorithm or structure that can be realized in the desired technology. Thus, the proposed finite memory residual generation filter is specified to the well known digital filter structure in [16] for the amenability to hardware implementation.

The filter gain matrix $\mathcal{H}_{s}$ for the $s^{\text {th }}$ residual $r_{s}(i)$ in (13) can be defined by

$$
\mathcal{H}_{s} \triangleq\left[h_{s}(M-1) h_{s}(M-2) \cdots h_{s}(0)\right]
$$

Then, the proposed residual generation filter for the $s^{\text {th }}$ residual $r_{s}(i)$ is given by

$$
r_{s}(i)=\sum_{j=0}^{M-1} h_{s}(j) y(i-j)-\sum_{j=0}^{M-1}\left[\mathcal{H}_{s} \Omega\right]_{j} u(i-j)
$$

where $\left[\mathcal{H}_{s} \Omega\right]_{j}$ is the $(j+1)^{t h} l$ elements of $\left[\mathcal{H}_{s} \Omega\right]$. Applying the $z$-transformation to the residual (16) yields the following digital filter structure:

$$
r_{s}(z)=\sum_{j=0}^{M-1} h_{s}(j) z^{-j} y(z)-\sum_{j=0}^{M-1}\left[\mathcal{H}_{s} \Omega\right]_{j} z^{-j} u(z)
$$

where $h_{s}(j)$ and $-\left[\mathcal{H}_{s} \Omega\right]_{j}$ become filter coefficients. It is noted that the digital filter structure (17) is a well known moving average process whose functional relation between inputs $y(z), u(z)$ and output $r_{s}(z)$ is nonrecursive. The block diagram of the proposed finite memory residual generation filter (12) for a hardware implementation can be represented as Fig. ??.

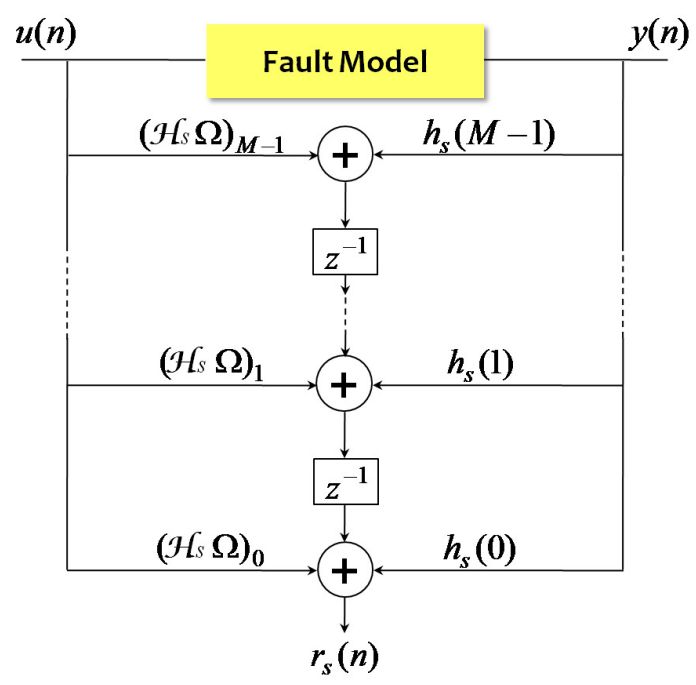

Figure 1. Block diagram representation of the finite memory residual generation filter 


\section{Computer Simulations}

To illustrate the capability of the proposed finite memory residual generation filter for a fault detection, numerical examples are performed for the following discrete-time DC motor system with the sensor fault:

$$
\begin{aligned}
x(i+1) & =\left[\begin{array}{cc}
-0.0005 & -0.0084 \\
0.0517 & 0.8069
\end{array}\right] x(i)+\left[\begin{array}{l}
0.1815 \\
1.7902
\end{array}\right] u(i)+\left[\begin{array}{l}
0.0006 \\
0.0057
\end{array}\right] w(i), \\
y(i) & =\left[\begin{array}{ll}
1 & 0 \\
0 & 1
\end{array}\right] x(i)+\left[\begin{array}{ll}
1 & 0 \\
0 & 1
\end{array}\right] f(i)+v(i) .
\end{aligned}
$$

Through computer simulations, the performance of the proposed finite memory residual generation filter is evaluated and compared with the Kalman filtering based residual generation filter with infinite memory structure in [4]-[9]. A fault is modeled for two scenarios as shown in Fig. 2. For the $1^{\text {st }}$ scenario, the fault is modeled as an incipient soft bias-type fault. In contrast, for the $2^{\text {nd }}$ scenario, the fault is modeled as an abrupt bias-type fault. The $2^{\text {nd }}$ scenario might be more feasible than the $1^{\text {st }}$ scenario. In these numerical examples, the window length is taken as $M=10$. Noise covariances are taken by $Q_{\omega}=0.01^{2}, Q_{\delta}=\operatorname{diag}\left(0.02^{2} 0.02^{2}\right)$ and $R=\operatorname{diag}\left(0.2^{2} 1^{2}\right)$.

The proposed finite memory residual generation filter is compared with the Kalman filtering based approach with infinite memory structure for both scenarios. Fig. 3 and 4 show plots for residuals of both residual generation filters. As shown in Figure 2 for the $1^{\text {st }}$ scenario, the proposed finite memory residual generation filter can be comparable to the Kalman filtering based approach for the incipient soft bias-type fault. In contrast, as shown in Figure 3 for the $2^{\text {nd }}$ scenario, the tracking of the proposed finite memory residual generation filter is much faster than the Kalman filtering based approach when the abrupt bias-type fault occurs and disappears. One possible explanation for this is the finite convergence time and fast tracking ability of the proposed finite memory residual generation filter.

\section{Concluding Remarks}

This paper has proposed a residual generation filter with finite memory structure for sensor fault detection. The proposed finite memory residual generation filter provides the residual by real-time filtering of fault vector using only the most recent finite measurements and inputs on the window. It has been shown that the residual given by the proposed residual generation filter provides the exact fault for noise-free systems. The proposed residual generation filter has been specified to the digital filter structure for the amenability to hardware implementation. Through numerical examples for the discretized DC motor system having the sensor fault. the capability of the proposed residual generation filter has been verified. 


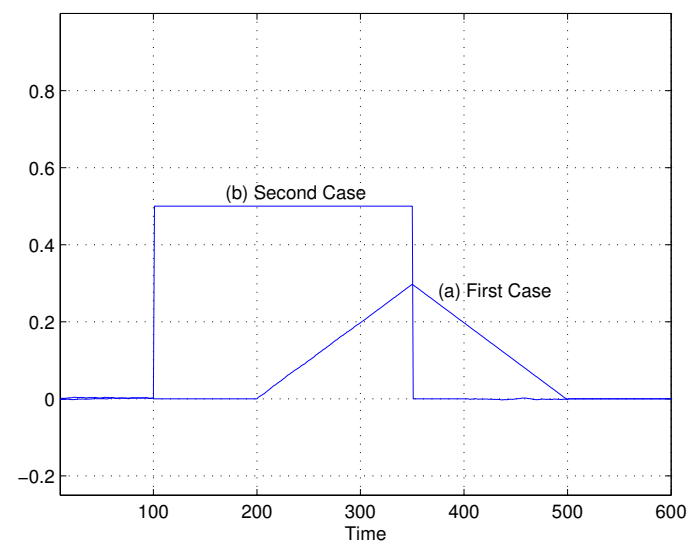

Figure 2. Two kinds of simulated faults

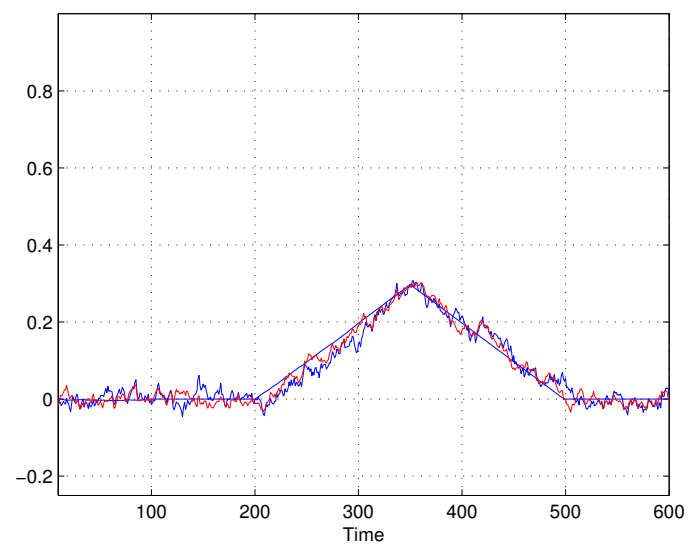

Figure 3. Residuals for the $1^{\text {st }}$ scenario

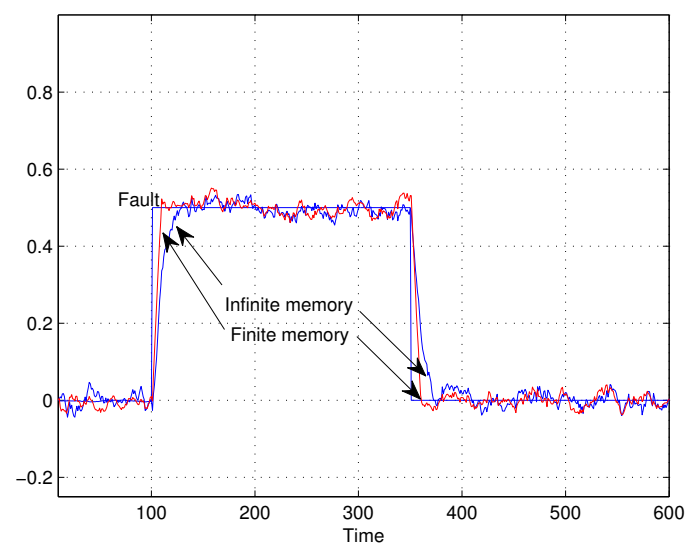

Figure 4. Residuals for the $2^{\text {nd }}$ scenario 
1. Venkatasubramanian, V.; Rengaswamy, R.; Yin, K.; Kavuri, S.N. A review of process fault detection and diagnosis - Part I: Quantitative model-based methods. Computers and Chemical Engineering 2003, 27, 293-311.

2. Angeli, C.; Chatzinikolaou, A. On-line fault detection techniques for technical systems: A survey. International Journal of Computer Science \& Applications 2004, 1, 12-30.

3. Hwang, I.; Kim, S.; Kim, Y.; Seah, C. A survey of fault detection, isolation, and reconfiguration methods. IEEE Trans. Control Systems Technology 2010, 18, 636-653.

4. Kobayashi, T.; Simon, D.L. Enhanced bank of Kalman filters developed and demonstrated for in-flight aircraft engine sensor fault diagnostics. Research and Technology, NASA Glenn Research Center at Lewis Field 2005, 2005-213419, 25-26.

5. Wang, Y.; Zheng, Y. Kalman filter based fault diagnosis of networked control system with white noise. Journal of Control Theory and Application 2005, 3, 55-59.

6. Tudoroiu, N.; Khorasani, K. Satellite fault diagnosis using a bank of interacting Kalman filters. IEEE Trans. Aerosp. Electron. Syst. 2007, 43, 1334-1350.

7. Xue, W.; Guo, Y.; Zhang, X. Application of a bank of Kalman filters and a robust Kalman filter for aircraft engine sensor/actuator fault diagnosis. International Journal of Innovative Computing, Information and Control 2008, 4, 3161-3168.

8. Tudoroiu, N. Real time embedded Kalman filter estimators for fault detection in a satellite's dynamics. International Journal of Computer Science $\mathcal{E}$ Applications 2011, 8, 83-109.

9. Villez, K.; Srinivasanb, B.; Rengaswamyb, R.; Narasimhanc, S.; Venkatasubramaniana, V. Kalman-based strategies for fault detection and identification (FDI): Extensions and critical evaluation for a buffer tank system. Computers and Chemical Engineering 2011, 35, 806-816.

10. Bruckstein, A.M.; Kailath, T. Recursive limited memory filtering and scattering theory. IEEE Trans. Inform. Theory 1985, 31, 440-443.

11. Kim, P.S. An alternative FIR filter for state estimation in discrete-time systems. Digital Signal Processing 2010, 20, 935-943.

12. Kim, P.S. A computationally efficient fixed-lag smoother using recent finite measurements. Measurement 2013, 46, 846-850.

13. Zhao, S.; Shmaliy, Y.S.; Huang, B.; Liu, F. Minimum variance unbiased FIR filter for discrete time-variant systems. Automatica 2015, 53, 355-361.

14. Pak, J.; Ahn, C.; Shmaliy, Y.; Lim, M. Improving reliability of particle filter-based localization in wireless sensor networks via hybrid particle/FIR filtering. IEEE Transactions on Industrial Informatics 2015, 11, 1-10.

15. Kim, P.S.; Jang, M.S.; Kang, S.Y.; Lee, E.H. A finite memory structure filtering for indoor positioning in wireless sensor networks with measurement delay. International Journal of Distributed Sensor Networks 2016, In press.

16. Oppenheim, A.; Schafer, R. Discrete-Time Signal Processing; Englewood Cliffs, NJ:Prentice-Hall, 1989.

(C) 2016 by the authors; licensee Preprints, Basel, Switzerland. This article is an open access article distributed under the terms and conditions of the Creative Commons Attribution (CC-BY) license (http://creativecommons.org/licenses/by/4.0/). 